and these data were used for the impact assessment. Some of the different impact categories included acidification, climate change, eutrophication, human health, land use, resource use and water scarcity.

The results concluded that the electric toothbrush had the greatest environmental impact in 15 out of the 16 categories with water scarcity being the exception. The plastic manual replaceable head and bamboo toothbrushes had the lowest impact in all categories. The results also showed how each stage of the product's life cycle contributed to the impact assessment. For example, regarding the manual plastic toothbrush and plastic manual replaceable head, the materials were the largest contributing factor; whereas for the electric toothbrush it was transport and for the bamboo manual toothbrush it was consumer use.
It is interesting to note that although an electric toothbrush is better for reducing plaque levels, the authors found no current evidence that their use reduces the incidence of dental caries or periodontal disease. This creates the question as to whether consumers will be able to switch to a more sustainable toothbrush, such as the bamboo toothbrush, without being at risk of developing more dental disease. It is clear from this study that further research is needed in the sustainability of toothbrushes. However, it is important to note that these results can be used as a tool to influence consumer choice by educating them regarding environmental sustainability.

Anika Shah

Department of Oral and Maxillofacial Surgery, Wexham Park Hospital

\title{
From face-to-face to face-time: is the future of dental appointments virtual?
}

Teledentistry from a patient perspective during the coronavirus pandemic. Br Dent J 2020; 229: http://dx.doi.org/10.1038/s41415-020-1919-6

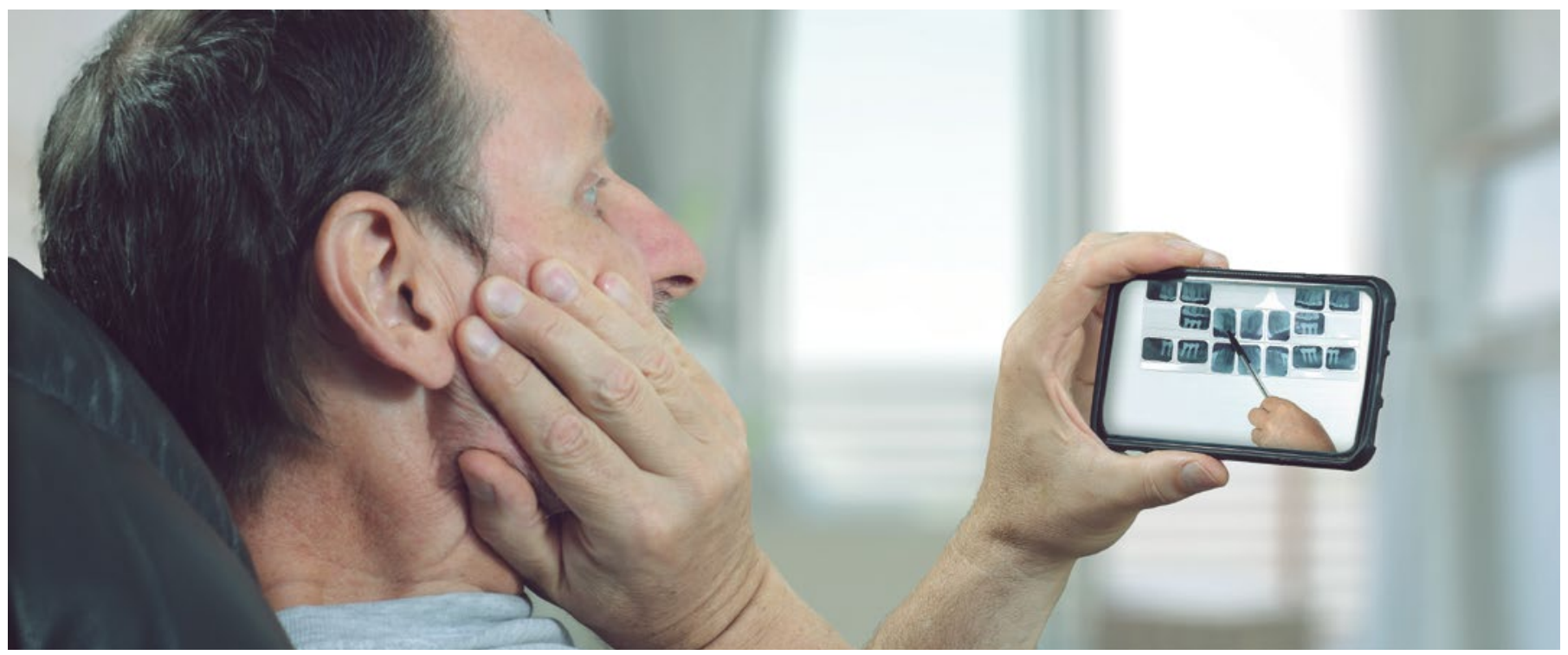

As dentists we are accustomed to being only centimetres away from our patients for long periods of time - this is the nature of our close contact profession. When the COVID-19 pandemic hit earlier this year, this unprecedented event undoubtedly changed the way in which the world works within a few short months, however, the consequences and changes that occurred are likely to last a lifetime, and dentistry is no exception to that. With practices forced into closure, dental teams had to quickly adapt to find a means of effective communication with their patients to discuss, diagnose and, where possible, treat the dental problems they were facing. Dental intervention generally requires the use of our tools for treatment, from forceps to high speeds, so how 列

The authors of this paper investigated patient perceptions of 'teledentistry' since the COVID-19 pandemic in East Surrey Hospital. Virtual clinics were introduced in the hospital in the forms of telephone and video consultations via NHS England's Attend Anywhere platform. The aim of 部 this was to allow some form of dentistry to continue while reducing the risk of viral transmission. At the end of each virtual session, participants were asked to complete a survey rating various aspects of the clinic such as access, usefulness and effectiveness. The 52 participants generally rated their virtual experiences highly in all aspects with over $90 \%$ agreeing to all statements and being willing to use the system again.

While the pandemic has brought about a time of uncertainty, the results from this study show that the dental community and its patients have been very quick to adapt to the 'new normal' and may encourage some welcome change to the way in which we work. With UDA targets, time pressure and volume of patients needing treatment, the conversation between dentists and patients has become muted over the past decade. The lack of time means appointments are used for necessary intervention rather than advice, prevention and behaviour changing conversations. This shift towards increased quality and quantity of communication with patients may help to shift the balance from intervention to prevention. This positive study highlights the ways we can improve our quality of patient care moving forwards in light of the COVID-19 pandemic.

Lorna Gladwin

DCT, Hull, UK 